\title{
ANTIEPILEPTIC DRUG POLYTHERAPY IN PREGNANT WOMEN WITH EPILEPSY
}

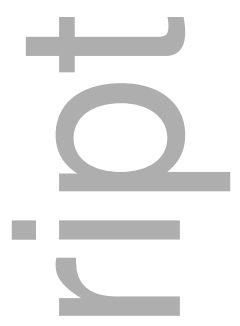

F J E Vajda ${ }^{\text {a }}$, T J O’Brien ${ }^{\text {a }}$, JE Graham ${ }^{\mathrm{a}}$, AA Hitchcock ${ }^{\mathrm{a}}$, C M Lander ${ }^{\mathrm{b}}$, M J Eadie ${ }^{\mathrm{b}}$

a Department of Medicine and Neurosciences, Royal Melbourne Hospital and University of Melbourne, Parkville, Victoria, Australia 3050

b Royal Brisbane and Women's Hospital and School of Medicine and Biomedical Science, University of Queensland, Brisbane, Queensland, Australia 4027

\section{Corresponding Author:}

Professor FJE Vajda

Address: Department of Medicine and Neurosciences, Royal Melbourne Hospital and University of Melbourne, Parkville, Australia 3050

Telephone: $\quad$ 61(3).98193056

E-mail: $\quad$ vajda@netspace.net.au

Word count: Abstract 151 words: Main text 3181 words; 2 Figs, 4 Tables

Running Title: Antiepileptic Drug Polytherapy in Pregnant Women

This is the author manuscript accepted for publication and has undergone full peer review but has not been through the copyediting, typesetting, pagination and proofreading process, which may lead to differences between this version and the Version of Record. Please cite this article as doi: 10.1111/ane.12965

This article is protected by copyright. All rights reserved 
PROF. FRANK VAJDA (Orcid ID : 0000-0001-5570-7538)

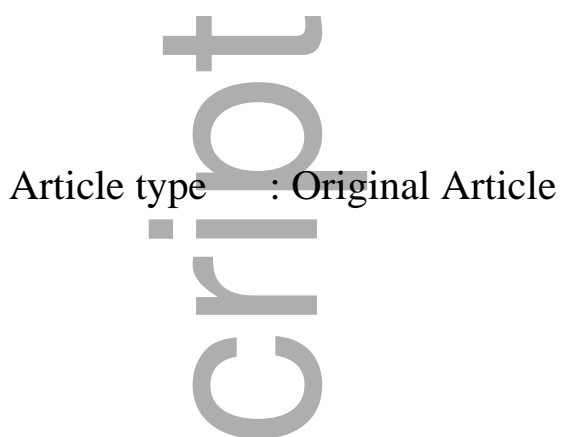

\section{Abstract}

Objective: To study seizure control and rates of foetal malformation in pregnancies of Women with epilepsy treated with antiepileptic drug polytherapy.

Methods: Use of conventional statistical methods to analyse the Australian Pregnancy Register records of 1810 pregnancies in women with epilepsy, 508 treated with antiepileptic drug polytherapy.

Results: $\quad$ Polytherapy-treated pregnancies were less often seizure free than monotherapy-treated ones, for both focal $(36.0 \%$ versus $51.9 \%$ : $\mathrm{P}<0.05)$ and primary generalised epilepsies $(41.1 \%$ versus $69.3 \%$; $\mathrm{P}<0.05)$. Drug combinations with dissimilar and similar mechanisms of action achieved similar rates of seizure freedom during pregnancy (36.3\% versus $38.3 \%)$. The increased rate of malformed foetuses in polytherapy pregnancies depended on valproate or topiramate being in the drug combinations. The combinations of lamotrigine and levetiracetam offered the chance of seizure control and foetal safety.

Conclusions In pregnancy, use of antiepileptic drug combinations is not necessarily disadvantageous to mother and foetus if valproate and topiramate are avoided. 
Key Wards: antiepileptic drugs; malformations; polytherapy; seizure control

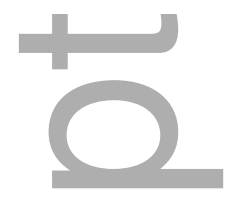

\section{$1 \quad$ Introduction}

The use of antiepileptic drug (AED) combinations began to be explored after the mid-20 $0^{\text {th }}$ century, when increasing numbers of reasonably effective and acceptably safe AEDs became available. Initially, AED polytherapy seems to have been prescribed mainly when a first drug appeared to fail to achieve seizure control ${ }^{1}$. Then, after plasma AED concentration monitoring began to be employed, evidence appeared that a single AED in appropriate dosage guided by AED concentration measurement would control seizures in a considerable majority of patients with newly diagnosed epilepsy ${ }^{2-4}$. It was therefore suggested that appropriately managed AED monotherapy, with its simpler pharmacokinetic situation and lessened variety of potential adverse effects, would usually provide effective management of epilepsy. This idea probably discouraged any expanded use of antiepileptic drug polytherapy, at least for a time. Nonetheless, in practice, instances remained where an optimally employed single AED failed to prevent further seizures, and AED polytherapy was employed. Unfortunately, AED combinations were shown to yield only a relatively small gain in seizure control in patients with focal epilepsies if a potentially appropriate first drug has already failed $^{5,6}$. The logical possibility was therefor raised that combinations of AEDs with different molecular mechanisms of action might provide a better chance of seizure control than combinations of AEDs all of which possessed the same mechanism of action, e.g. on $\mathrm{Na}^{+}$ channels, $\mathrm{GABA}-\mathrm{Cl}^{-}$or synaptic vesicle mechanisms, AMPA receptors ${ }^{1,7}$. However, few studies are yet available demonstrating that this expectation applies in practice ${ }^{8}$.

In women with epilepsy, some earlier studies have suggested that there is a heightened risk of foetal malformation in pregnancies exposed to AED combinations ${ }^{9-15}$, though this was not the case in an earlier analysis of Australian Pregnancy Register (APR) data ${ }^{16}$. 
It therefore seemed worth studying the simultaneous foetal malformation and seizure control outcomes associated with AED polytherapy in the pregnant women with epilepsy whose data have been collected in the APR over the past 18 years,

\section{$2 \quad$ Materials and methods}

Since 1998, the Australian Pregnancy Register (APR) has collected data on the pregnancies of Australian women who (i) are taking AEDs, nearly always for epilepsy, (ii) did not take these drugs in at least the first half of pregnancy. More detailed information regarding the APR and its recruitment policies and data handling practices has been published previously ${ }^{17,18}$. Once women know of the Register's existence, whether by professionals referring them to it, or by personal enquiry or lay suggestion, they may choose whether to be included in its database, which has been estimated to contain information on about $8.7 \%$ of the relevant pregnancies in Australia ${ }^{19}$. All contact throughout between pregnant women and the APR is via telephone. Data concerning each woman's pre-pregnancy epilepsy situation and the course of her pregnancy are recorded (i) at enrolment, (ii) at approximately 28 weeks of pregnancy, (iii) within the first post-partum month and (iv) as far as possible, at one year after giving birth (sometimes it has proved impossible to contact women after that interval and a few pregnancies are too recent to obtain one-year data). The accuracy of the information provided by pregnant women is checked with their treating medical practitioners. Throughout pregnancy, the clinical management of pregnancy always remains in the hands of the treating medical practitioners. The APR does not attempt to influence that management. Over the years the APR has been under the ethics oversight of various Melbourne based institutional ethics committees, that of the University of Melbourne currently holding responsibility.

The foetal malformations considered were structural ones recognised during pregnancy, at or soon after birth and in the first year of post-natal life, and were judged significant enough by those reporting them to warrant correction measures, if feasible. Multiple malformations occurred in some pregnancies, but the present analysis is in terms of numbers of pregnancies with malformed foetuses, and not numbers of individual malformations.

For the present study, relevant data from the APR were transferred to an Excel spreadsheet and then analysed, employing confidence interval methods and linear or logistic regression techniques, as appropriate. 


\section{$3 \quad$ Results}

\subsection{The pregnancies studied}

The present study was based on the records of 2089 pregnancies contained in the APR at mid-2017. After exclusion of pregnancies in which antiepileptic drugs were not taken throughout the whole of pregnancy, or which ended prematurely (mainly due to spontaneous abortion) or where antiepileptic drugs were taken for indications other than epilepsy, there remained 1810 pregnancies in 1403 women with epilepsy who took AEDs throughout the whole course of pregnancy. Different pregnancies in the same woman had sometimes been managed in one pregnancy with AED monotherapy, and in another with polytherapy, so that the data had to be analysed in relation to pregnancies rather than women. There were another 162 pregnancies in women with epilepsy who had not taken AEDs during at least the initial four months of pregnancy, but these were excluded from analysis except for comparative purposes in relation to foetal abnormality rates.

AED combinations were taken throughout pregnancy in 508 of the 1810 pregnancies studied (28.1\%). AED monotherapy was involved in the remaining $71.9 \%$.

The mean ages at which pregnancies commenced in the polytherapy and monotherapy pregnancies did not differ statistically significantly (respectively, $30.47 \pm 4.84$ and $31.01 \pm$ 4.51 years: difference $=-0.54$ years; $95 \%$ C.I. $-1.01,0.07$ years). The mean duration of epilepsy prior to pregnancy was longer in the polytherapy pregnancies $(16.46 \pm 8.21$ versus $13.15 \pm 8.34$ years; difference $=3.31$ years; $95 \%$ C.I. $2.46,4.16$ years $)$, probably because of years of failed AED monotherapy before polytherapy was commenced. The polytherapy pregnancies were associated with a shorter mean seizure-free interval prior to pregnancy than the monotherapy ones $(1.64 \pm 3.1$ versus $2.93 \pm 3.9$ years; difference $=1.28$ years; $95 \%$ C.I. $0.91,1.65$ years $)$.

The type of seizure disorder involved could be determined in 1671 of the 1810 pregnancies. Of the latter 1810, 49.0\% had focal epilepsies and $43.3 \%$ generalised ones. Focal epilepsies accounted for $55.6 \%$ and generalised epilepsies for $44.4 \%$ of the 482 polytherapy pregnancies with known types of seizure disorder. For the 1189 monotherapy pregnancies, the corresponding figures were $52.1 \%$ and $47.9 \%$.

\subsection{The AED combinations used}


Two AEDs were combined in $82.3 \%$ of the polytherapy pregnancies, three in $14.1 \%$, four in $2.8 \%$ and five in $0.8 \%$. There was a non-statistically significant trend to increasing use of AED polytherapy across the study period. Such use involved $26.2 \%$ of pregnancies in the 1998-2000 triennium (by date of last menstrual period), $24.2 \%$ in the $2001-2003$ one, $29.6 \%$ in $2004-2006,23.3 \%$ in $2007-2009,35.6 \%$ in $2010-2012$ and $31.8 \%$ in the final triennium (an increase annual rate in linear regression analysis of $0.53 \% ; \mathrm{P}=0.18$ ).

Lamotrigine (LTG, in 51.6\%) was the most frequently used AED in polytherapy, followed by carbamazepine (CBZ) and valproate (VPA), each in 35.0\%, levetiracetam (LEV) in $27.6 \%$, topiramate (TPM) in $18.3 \%$, clonazepam (CZP) in $17.1 \%$, phenytoin (PHT) in $7.1 \%$, gabapentin (GPT) in 3.9\% and oxcarbazepine (OXC) in 2.4\%. Various other AEDs were used occasionally. Figure 1, middle panel shows the changing patterns of use of the more frequently employed individual drugs in the polytherapy pregnancies between 1998 and 2016. There was a progressive decline in the use of older antiepileptic drugs such as valproate, carbamazepine, phenytoin, clonazepam, with increasing use of levetiracetam and topiramate. There was a similar usage trend for the same individual AEDs in monotherapy (Figure 1 top panel but it lagged some 3 years behind the polytherapy pregnancies. Probably the newer drugs had been tried in polytherapy in difficult-to-control epilepsies before prescriber confidence in their usefulness and safety grew sufficiently for their use in monotherapy.

Numerous combinations of individual AEDs had been employed. The more frequent ones are shown in Table 1.

\subsection{Seizure control}

Seizures were fully controlled throughout pregnancy and labour in 38.2\% of polytherapy pregnancies and $60.1 \%$ of monotherapy ones (O.R. $=0.41 ; 95 \%$ C.I. $=0.33,0.51)$. After excluding pregnancies where seizure disorders had begun less than a year before the last menstrual period, the pre-pregnancy year was seizure-free in $42.0 \%$ of 481 polytherapy pregnancies, and in $63.5 \%$ of 1175 monotherapy ones (O.R. $=0.42,95 \%$ C.I. $=0.34,0.52)$. Seizure freedom throughout pregnancy and labour occurred in $38.3 \%$ of the polytherapy pregnancies and in $60.3 \%$ of the corresponding monotherapy ones $(\mathrm{O} . \mathrm{R} .=0.41,95 \%$ C.I. $=$ $0.33,0.1)$. 
Table 2 shows the percentages of pregnancies with full seizure control separately for focal and generalised epilepsy in pregnancies exposed to AED polytherapy and monotherapy

In the polytherapy pregnancies, seizure control was worse in the pre-pregnancy year and throughout pregnancy and labour. This applied for both focal and generalised epilepsies. The lowest panel in Figure 1 shows an apparent trend towards increased seizure freedom during pregnancy as time passed, particularly in the polytherapy pregnancies, but the trend in linear regression (1.18\% per annum) was not statistically significant $(\mathrm{P}=0.07)$.

\subsubsection{Rational AED combinations}

AED combinations restricted to drugs with similar molecular mechanisms of antiepileptic action had been used in 274 of the 508 polytherapy pregnancies. At least two AEDs with dissimilar action mechanisms had been employed in the remaining 234 polytherapy pregnancies. In the subgroup taking AEDs with similar action mechanisms, 114 pregnancies (41.6\%) had been seizure free for at least a year before pregnancy: for the dissimilar subgroup the corresponding number was 92 (39.3\%). Seizure freedom throughout pregnancy and labour had occurred in $38.3 \%$ of the similar mechanisms subgroup, and in $36.3 \%$ of the dissimilar mechanisms subgroup. Thus in the population studied there was little to suggest that combining antiepileptic drugs with different mechanisms of molecular action had been associated with better seizure control.

\subsection{Foetal Malformations}

Foetal malformations had occurred in 44 of the 508 polytherapy pregnancies $(8.7 \%)$ and in 87 of the 1302 monotherapy pregnancies $(6.7 \%)$. There had also been five pregnancies with foetal malformations in the 162 pregnancies in women with epilepsy (3.1\%) who had not taken antiepileptic drugs in at least the first half of their pregnancies. The malformed pregnancy rate was statistically significantly higher in the polytherapy pregnancies than in the AED unexposed ones (O.R. $=2.98,95 \%$ C.I. 1.16, 7.64), but not so in the monotherapy ones $(\mathrm{O} . \mathrm{R} .=2.25,95 \%$ C.I. $0.90,5.62)$. Nor was the malformed pregnancy rate higher at a $\mathrm{P}<0.05$ level in the polytherapy pregnancies than in the monotherapy ones (O.R. $=1.32$, 95\% C.I. $0.91,1.93)$.

Earlier studies based on a smaller cohort from the APR had shown that the foetal malformation rates were drug dosage related, at least for valproate exposure ${ }^{20-22}$ and topiramate, but only in polytherapy for the latter drug ${ }^{23}$. In the present data set, the mean 
valproate dose was $859 \pm 635 \mathrm{mg}$ per day in the polytherapy pregnancies and $904 \pm 574 \mathrm{mg}$ per day in the monotherapy ones. For topiramate dosage the corresponding numbers were 246 \pm 138 and $196 \pm 118 \mathrm{mg}$ per day. The difference in mean valproate doses between the two populations was not statistically significant, but the difference in mean topiramate dose was. There were also statistically significant differences in mean carbamazepine and levetiracetam dosages between the polytherapy and monotherapy groups. Logistic regressions were calculated for risk of pregnancy with malformations on AED dosage for the more commonly employed AEDs in the polytherapy and monotherapy pregnancies (Table 3). Statistically significant dose-related increases in foetal malformation risk were found only for valproate and topiramate in the polytherapy pregnancies, and a dose-related decrease in risk for lamotrigine. In the monotherapy pregnancy group there was a dose-related increase in risk for pregnancy with malformation only for valproate.

The regression lines for malformation risk on valproate dosage showed increasing divergence between the monotherapy and polytherapy populations studied as the drug dose increased The directions of slope for the regression lines for malformation risk on topiramate dosage differed (Figure 2), malformation risk increasing with increasing topiramate dose in the polytherapy subgroup, while there being no statistically significant regression for the monotherapy pregnancies.

There had been 15 malformed foetuses in 93 pregnancies exposed to topiramate in polytherapy $(16,1 \%), 3$ occurring in combinations with carbamazepine (10\%) 2 with valproate (13\%), 2 with lamotrigine (7\%), 5 with levetiracetam (20\%), and single instances with phenytoin, clonazepam and pregabalin. This information does not provide any obvious explanation for the dose-dependent association between topiramate in polytherapy and foetal malformation.

The role of polytherapy per se in foetal malformation was assessed, malformation rates being recalculated, as separate exercises, for the polytherapy and monotherapy groups following exclusion of pregnancies exposed to valproate and to topiramate. With valproate-exposed pregnancies excluded, the malformed foetus rates became 8.18\% (27 in 330) for polytherapy pregnancies, and $4.77 \%$ (87 in 1007) for monotherapy ones (O.R. $=1.78,95 \%$ C.I. 1.09, 2.90). Excluding only topiramate-exposed pregnancies yielded malformation rates of $7.00 \%$ (29 in 415) for polytherapy pregnancies and 6.87\% (86 in 1252) for monotherapy pregnancies $(\mathrm{O} . \mathrm{R} .=1.02,95 \%$ C.I. $0.66,1.58)$. The apparent higher incidence of foetal malformations in 
the polytherapy pregnancy group as compared with the monotherapy one appeared to relate to pregnancies exposed to topiramate, rather than to AED polytherapy per se.

\subsection{Desirable AED Combinations}

The most desirable outcome of managing pregnancy in women with epilepsy is the birth of a normal foetus after a seizure-free pregnancy. The rate of having achieved this outcome in the polytherapy pregnancies is shown in Table 4 . The pairing of lamotrigine and levetiracetam most nearly approached the desired situation. In monotherapy pregnancies, lamotrigine had been associated with a $4.7 \%$ foetal malformation rate and a seizure freedom rate during pregnancy of $51.0 \%$, and levetiracetam with a malformation rate of $3.1 \%$ (the same rate as that in the pregnant women not exposed to AEDs throughout), and a pregnancy seizure freedom rate of $67.1 \%$.

\section{Discussion}

We are not aware of a previous study that has examined both seizure control and foetal malformation rates in the same set of pregnancies in women with epilepsy treated with AED polytherapy. However, the present study has limitations. It is based on not a random sample drawn from the whole relevant population but on a subset of pregnancies that may be biased by preferential self-inclusion by women who are concerned with their own and their foetus's well-being, and possibly by women with more difficult-to-control epilepsies (nearly half of the pregnancies were referred to the APR by neurologists). The data for the pre-pregnancy seizure freedom rates have necessarily been collected retrospectively, unlike the data for the pregnancies themselves. The drugs employed in the individual drug combinations were not allocated according to any deliberate pattern, simply being prescribed as treating medical practitioners saw fit. To some extent these patterns of prescribing may have been influenced by previously published malformation hazard findings derived from the Australian Register. Many individual drug combinations had been employed, some in only a few pregnancies, so that conclusions drawn from the less frequent combinations may be unreliable. It was not practicable for women to keep seizure diaries or counts of minor seizure, so that the only criterion of epilepsy control that could be studied reliably was freedom from seizures, though this usually is the crucial issue in managing epilepsy. Foetal malformations could be considered only as an overall class. There were too few instances of specific malformations, 
and sometimes more than one malformation in relation to an individual pregnancy, for any simple analysis.

Nevertheless, several reasonably reliable conclusions can be drawn from the study. AED polytherapy seems to have been employed in most of the women studied because previous AED monotherapy had proved unsatisfactory. The explicit reason for employing polytherapy was not recorded in the Register, but the fact that the polytherapy population had a longer mean duration of seizure disorder than the monotherapy population, despite similar mean ages at onset of pregnancy, had a shorter mean duration of seizure freedom prior to pregnancy, and had a lower rate of freedom from seizures during pregnancy, are consistent with this interpretation.

The study provided no evidence that pharmacologically rational antiepileptic drug combinations involving agents with different molecular mechanisms of action yielded a better chance of seizure freedom during pregnancy than combinations of drugs with similar molecular mechanisms of action. The rationale underlying the anticipation of benefit from this approach depends on the initial AED used having been taken to its maximum tolerated effect before another drug is added, and the Register did not trace information back far enough to ensure that this requirement had been met.

The findings of the present study, like those of several reasonably recent investigations ${ }^{16,24-27}$ do not support the earlier published belief that AED polytherapy in its own right increases the hazard of foetal malformation. The newer evidence has indicated that the heightened malformation risk with AED polytherapy depends on the presence of valproate in AED combinations, while the present data suggest that topiramate, in polytherapy, may have a similar effect. The information available to the present study provides no obvious explanation for this finding in relation to topiramate. It has been suggested that some spontaneous abortions may represent consequences of foetal malformations incompatible with extrauterine life ${ }^{28}$. Pregnancies ending in spontaneous abortion were excluded from the present analysis but, even so, an earlier study of APR material had found that AED polytherapy, as compared with monotherapy, was not associated with an increased risk of intrauterine foetal death ${ }^{29}$.

Those managing pregnancy in women with epilepsy would usually aim to minimise the risk of foetal malformation while maintaining freedom from seizures during pregnancy. No polytherapy combination studied in the present paper consistently achieved this desirable situation. The best approximation to it was achieved by pairing lamotrigine and 
levetiracetam. This knowledge may be useful when prescribing AEDs for women intending to become pregnant and still needing AED therapy. At the initiation of their therapy only a single AED would probably be employed. Based on the monotherapy data mentioned above, levetiracetam usually might appear the best initial choice, being associated with greater foetal safety and seizure control efficacy than lamotrigine. Then, if levetiracetam in maximum tolerated dosage failed to achieve seizure control. lamotrigine might be the best AED to add to it.

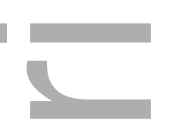

\section{Acknowledgements}

Professional and lay colleagues are thanked for referring patients to the APR, and the Scientific Advisory Board and the Ethical Research Committees of St. Vincent's Hospital, Monash Medical Centre, the Royal Melbourne Hospital and other institutions are thanked for continuing ethics oversight of the APR. Over the years, The Epilepsy Society of Australia, The Royal Melbourne Hospital Neuroscience Foundation, Epilepsy Australia, the NHMRC and the pharmaceutical companies Sanofi-Aventis, UCB Pharma, Janssen-Cilag, Novartis, Sci-Gen, Eisai and Genzyme have provided financial support for the Register

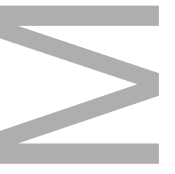

\section{Disclosure of conflict of interest}

F J E Vajda has received research support for the Australian Pregnancy Register from the Epilepsy Society of Australia, RMH Neuroscience Foundation, Epilepsy Action, SanofiAventis, UCB Pharma, Eisai, and Sci-Gen.

T O'Brien has received research support from the Epilepsy Society of Australia, NHMRC, RMH Neuroscience Foundation, Sanofi-Aventis, UCB Pharma, Eisai and Sci-Gen C M Lander, JE Graham, AA Hitchcock and M J Eadie have no relevant conflicts of interest to declare. No funding from outside bodies has been involved in their roles in this paper. 


\section{References}

1. Brodie MJ, Sills GJ. Combining antiepileptic drugs -rational polytherapy. Seizure 2011; 20:369-375.

2. Reynolds EH, Chadwick D, Galbraith AW. One drug (phenytoin) in the treatment of epilepsy. Lancet 1976; 307: 923-6

3. Shorvon SD, Reynolds EH. Reduction in polypharmacy for epilepsy. Br Med J 1979; 2:1023-1025.

4. Reynolds EH, Shorvon SD. Monotherapy or polytherapy for epilepsy? Epilepsia $1981 ; 22: 1-10$.

5. Schmidt D. Two antiepileptic drugs for intractable epilepsy with complex partial seizures. J Neurol Neurosurg Psychiatry 45:1119-1124

6. Schmidt D, Gram L. Monotherapy versus polytherapy in epilepsy. A reappraisal. CNS Drugs 1995; 3:194-208.

7. Kwan P, Brodie MJ. Epilepsy after the first drug fails: substitution or add-on? Seizure 2000; 9: 464-468.

8. Margolis JM, Chu BC, Wang ZJ, Copher R, Cavazos JE. Effectiveness of antiepileptic drug combination therapy for partial-onset seizures based on mechanisms of action. AMA Neurology 2014; 71: 985-993.

9. Nakane Y, Okuma T, Takahashi R et al. Multi-institutional study on the teratogenicity and fetal toxicity of antiepileptic drugs: a report of a collaborative study group in japan. Epilepsia 1980; 21: 663-680.

10. Kaneko S, Utami K, Kondo T et al. Malformations in infants of mothers with epilepsy receiving antiepileptic drugs. Neurology 1992; 42 (suppl 5): 68-74

11. Lindhout D, Omtzigt J G. Pregnancy and the risk of teratogenicity. Epilepsia 1992; 33 (suppl 4): S41-S48.

12. Samrén EB, van Duijn CM, Koch $S$ et al. Maternal use of antiepileptic drugs and the risk of major congenital malformations: a joint European prospective study of human teratogenesis associated with maternal epilepsy. Epilepsia 1997; 38:981-990.

13. Holmes LB, Harvey GA, Coull BA, Huntington KB, Khoshbin S, Hayes AM, Ryan LM. The teratogenicity of antiepileptic drugs. N Eng J Med 2001; 344: 1132-1138. 
14. Morrow J, Russell A, Guthrie E et al. Malformation risk of antiepileptic drugs in pregnancy: a prospective study from the UK Epilepsy and Pregnancy Register. J Neurol Neurosurg Psychiatry 2006; 77:193-198.

15. Wlodarczyk BJ, Palacios AM, George TM, Finnell RH. Antiepileptic drugs and pregnancy outcomes. Am J Med Genet A 2012; 158A: 2071-2090.

16. Vajda F, Hitchcock A, Graham J, O'Brien T, Lander C, Eadie M. The teratogenic risk of antiepileptic drug polytherapy. Epilepsia 2010; 51:805-810.

17. Vajda FJE, O’Brien T, Hitchcock A, Graham J, Lander C, Eadie M. The Australian AED in pregnancy register: aspects of data collection and analysis. J Clin Neurosci 2007; 14: 936-42.

18. Vajda, FJE, Hollingworth S, Graham J, Hitchcock AA, O’Brien, TJ, Lander CM, Eadie MJ. Changing patterns of AED use in pregnant Australian women. Acta Neurol Scand 2010; 121: 89-93.

19. Vajda FJE, O'Brien TJ, Graham, J, Lander CM, Eadie MJ. The Australian register of AEDs in pregnancy - changes with time in enrolled women with epilepsy. J Clin Neurosci 2013; 21:1478-1482.

20. Vajda FJE, Eadie MJ. Maternal valproate dosage and foetal malformations. Acta Neurol Scand 2005; 112:137-143.

21. Vajda FJE, Hitchcock A, Graham J, O’Brien T, Lander C, Eadie M The Australian Register of Antiepileptic Drugs in Pregnancy: the first 1002 pregnancies. Aust N Z J Obstet Gynaec 2007; 47: 468-474.

22. Vajda FJE, O'Brien TJ, Graham J, Lander CM, Eadie MJ. Dose-dependency of fetal malformations associated with valproate. Neurology 2013; 81:999-1003

23. Vajda F, O'Brien T, Graham J, Lander C, Eadie M. Antiepileptic drug combinations not involving valproate and the risk of fetal malformation. Epilepsia 2016; 57: 1048 1052.

24. Artama M, Auvinen A, Raudaskoski T, Isojarvi I, Isojarvi J. Antiepileptic drug use of women with epilepsy and congenital malformations in the offspring. Neurology 2005; 64:1874-1878.

25. Mawer G, Briggs M, Baker GA et al. Pregnancy with epilepsy: obstetric and neonatal outcome of a controlled study. Seizure 2010; 19:112-119.

26. Holmes LB, Mittendorf R, Shen A, Smith CR, Hernandez-Diaz S. Fetal effects of anticonvulsant polytherapies. Arch Neurol 2011; 68:1275-1281. 
27. Tomson T, Battino D, Bonizzoni E et al. 2015. Dose-dependent teratogenicity of valproate in mono- and polytherapy. Neurology 2015; 85: 866-872.

28. Vajda FJE, O'Brien TJ, Graham J, Hitchcock AA, Lander CM, Eadie MJ. Anti-epileptic drug exposure and risk of foetal death in utero. Acta Neurol Scand. 2018;137: 20-23.

29. Vajda FJ, O'Brien TJ, Graham JE, Hitchcock AA, Lander CM, Eadie MJ. Antiepileptic drugs, foetal malformations and spontaneous abortions. Acta Neurol Scand. 2017; 135: 360-365.

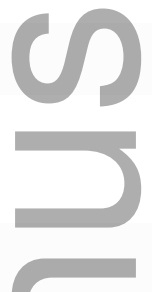

Table 1 . The more commonly employed pairs of antiepileptic drugs in all the 508 polytherapy pregnancies, and in the 416 polytherapy pregnancies that involved 2 drugs only.

Abbreviations: LTG=lamotrigine, VPA=valproate, $\mathrm{LEV}=$ levetiracetam, $\mathrm{CBZ}=$ carbamazepine, $\mathrm{CZP}=$ clonazepam, $\mathrm{TPM}=$ topiramate .

\begin{tabular}{lcccc}
\hline Combination & \multicolumn{2}{c}{ All Polytherapy } & \multicolumn{2}{c}{ Two AED Polytherapy } \\
\cline { 2 - 5 } & Number & \% & Number & \% \\
\hline LTG + VPA & 104 & 20.5 & 79 & 19.0 \\
LTG + CBZ & 51 & 10.0 & 39 & 9.4 \\
LTG + LEV & 50 & 9.8 & 36 & 8.7 \\
CBZ + LEV & 40 & 7.9 & 30 & 7.2 \\
LTG + CZP & 33 & 6.5 & 15 & 3.6 \\
CBZ + TPM & 30 & 5.9 & 13 & 3.1 \\
LTG + TPM & 28 & 5.5 & 18 & 4.3 \\
CBZ + VPA & 27 & 5.3 & 17 & 4.1 \\
CBZ + CZP & 26 & 5.1 & 14 & 3.4 \\
VPA + CZP & 26 & 5.1 & 13 & 3.1 \\
LEV + TPM & 25 & 4.9 & 14 & 3.4
\end{tabular}




$\begin{array}{lllll}\text { VPA + LEV } & 25 & 4.9 & 10 & 2.4\end{array}$

Table 2. Seizure freedom rates in the pre-pregnancy year and throughout pregnancy and labour for women with focal and generalised epilepsy who were managed with AED monotherapy or polytherapy than the monotherapy pregnancies in all 4 comparisons

\begin{tabular}{|c|c|c|c|c|}
\hline Epilepsy type & \multicolumn{2}{|c|}{ Focal } & \multicolumn{2}{|c|}{ Generalised } \\
\hline Number $(\%$ & \multicolumn{2}{|c|}{$887(53.1 \%)$} & \multicolumn{2}{|c|}{$784(46.9 \%)$} \\
\hline Treatment & Polytherapy & Monotherapy & Polytherapy & Monotherapy \\
\hline \multicolumn{5}{|c|}{ Throughout pre-pregnancy year } \\
\hline Number & 267 & 619 & 214 & 570 \\
\hline Seizure-free & 105 & 349 & 97 & 399 \\
\hline Percent & 39.3 & 56.9 & 49.3 & 70.7 \\
\hline \multicolumn{5}{|c|}{ Throughout pregnancy and labour } \\
\hline Seizure-free & 96 & 318 & 88 & 391 \\
\hline Percent & 36.0 & 51.9 & 41.1 & 69.3 \\
\hline
\end{tabular}

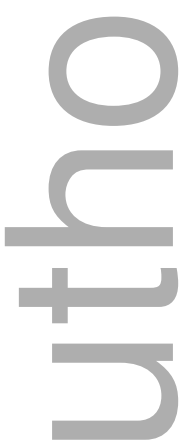

Table 3. Intercept values and coefficients (b) for logistic regressions for risk of foetal malformation on AED dose: logit risk $=a+b_{1} \cdot x_{1}+b_{2} \cdot x_{2}+\ldots b_{n} \cdot x_{n}$, where $b$ refers to each individual AED and $\mathrm{x}$ represents its dose.

\section{Polytherapy}




\begin{tabular}{lcccc} 
& \multicolumn{3}{c}{$\mathrm{P}=$} & $\mathrm{P}=$ \\
\hline Intercept value & -2.256599 & $<0.0001$ & -2.90731 & $<0.0001$ \\
Coefficients & & & & \\
Carbamazepine & -0.000689 & 0.1393 & +0.000537 & 0.1620 \\
Valproate & +0.000502 & 0.0491 & +0.001093 & $<0.0001$ \\
Lamotrigine & -0.002458 & 0.0293 & -0.000971 & 0.3733 \\
Levetiracetam & +0.000042 & 0.7957 & -0.000484 & 0.2178 \\
Topiramate & +0.003257 & 0.0042 & -0.003952 & 0.4301 \\
Phenytoin & -0.000948 & 0.6376 & -0.002462 & 0.4365 \\
Oxcarbazepine & -0.000717 & 0.6075 & +0.000603 & 0.4171 \\
Clonazepam & -0.099615 & 0.6397 & - & - \\
\hline
\end{tabular}

Table 4: Percentages for (i) foetal malformation rates and (ii) seizure-free pregnancies in the (a) pre-pregnancy year and (b) during pregnancy in all polytherapy pregnancies and in polytherapy pregnancies involving 2 AEDs only. Numbers of relevant pregnancies are shown in Table 1.

\begin{tabular}{|c|c|c|c|c|c|c|}
\hline \multirow{3}{*}{ Combination } & \multicolumn{3}{|c|}{ All Pregnancies } & \multicolumn{3}{|c|}{ Pregnancies with 2 AEDs only } \\
\hline & \multirow[t]{2}{*}{ Malformed } & \multicolumn{2}{|c|}{ Seizure free } & \multirow[t]{2}{*}{ Malformed } & \multicolumn{2}{|c|}{ Seizure free } \\
\hline & & Before & During & & Before & During \\
\hline $\mathrm{LTG}+\mathrm{VPA}$ & 8 & 46 & 41 & 10 & 53 & 43 \\
\hline $\mathrm{LTG}+\mathrm{CBZ}$ & 6 & 33 & 33 & 5 & 33 & 33 \\
\hline LTG + LEV & $\mathbf{0}$ & 50 & 46 & $\mathbf{0}$ & 53 & 47 \\
\hline $\mathrm{CBZ}+\mathrm{LEV}$ & 5 & 43 & 30 & 7 & 44 & 30 \\
\hline $\mathrm{LTG}+\mathrm{CZP}$ & 3 & 21 & 18 & 7 & 27 & 27 \\
\hline $\mathrm{CBZ}+\mathrm{TPM}$ & 10 & 27 & 20 & 23 & 46 & 46 \\
\hline $\mathrm{LTG}+\mathrm{TPM}$ & 7 & 18 & 21 & 11 & 22 & 22 \\
\hline
\end{tabular}




\begin{tabular}{lcccccc} 
CBZ + VPA & 15 & 33 & 22 & 23 & 35 & 24 \\
CBZ + CZP & 0 & 35 & 35 & 0 & 50 & 50 \\
VPA + CZP & 12 & 39 & 35 & 23 & 54 & 31 \\
LEV + TPM & 0 & 28 & 36 & 0 & 29 & 43 \\
VPA + LEV & 20 & 28 & 20 & 40 & 30 & 20 \\
\hline
\end{tabular}

口

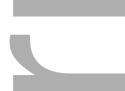

0

$\infty$
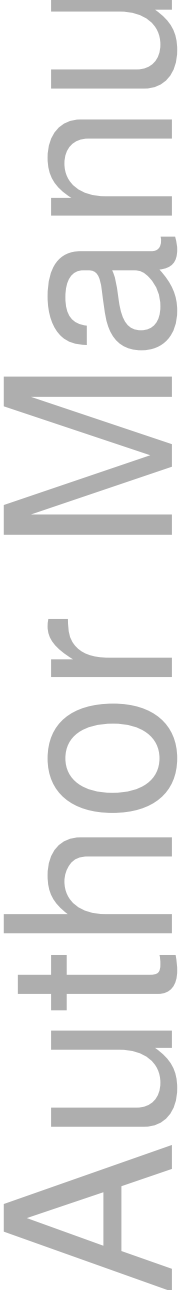

This article is protected by copyright. All rights reserved 

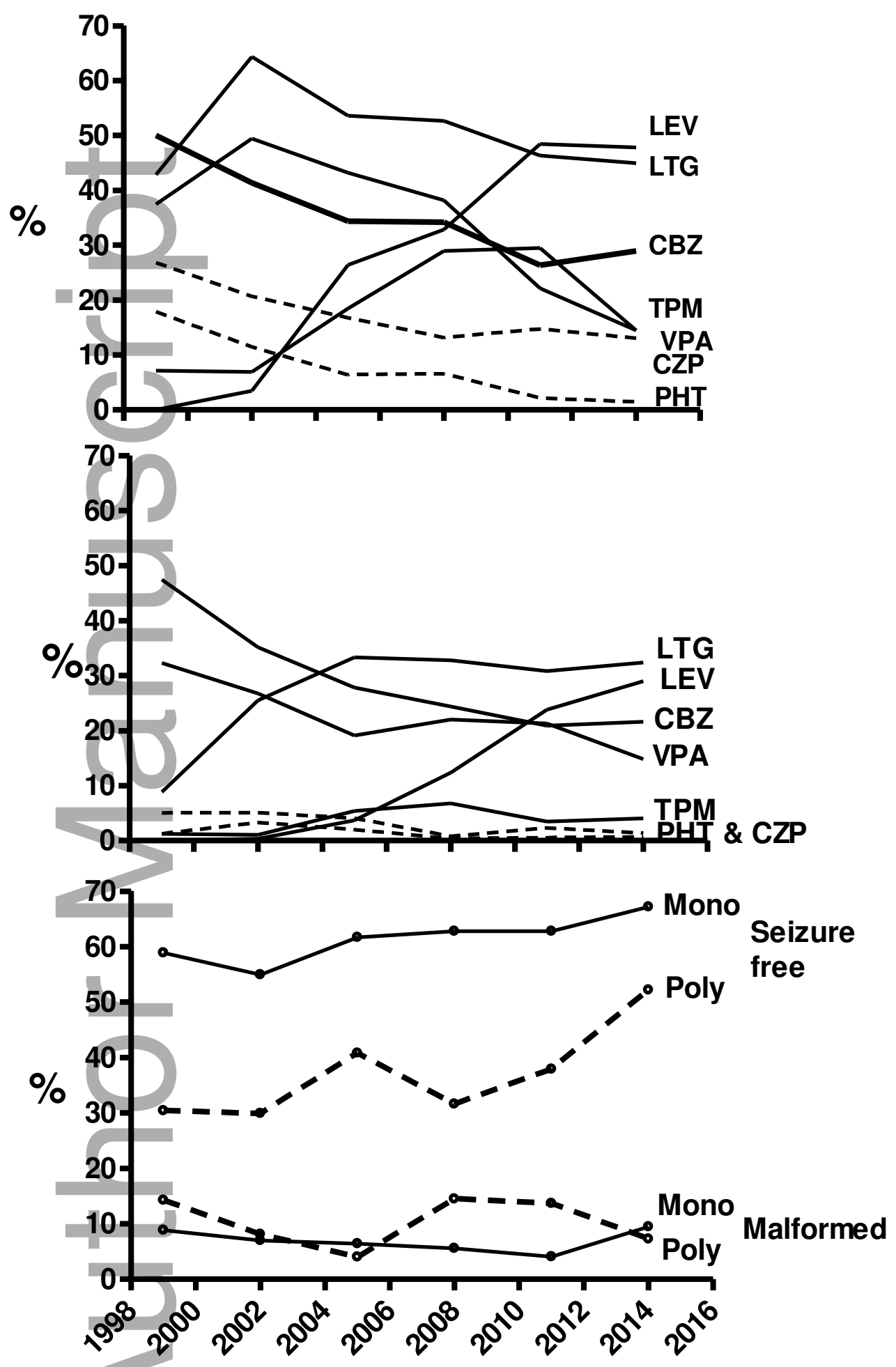

Fig 1. Top panel: Changing patterns of individual AED use in monotherapy with time Middle panel: Changing patterns of individual AED use in polytherapy with time 
Lower panel: Changing rates of seizure freedom and foetal malformation occurrence with time in monotherapy and polytherapy treated pregnancies
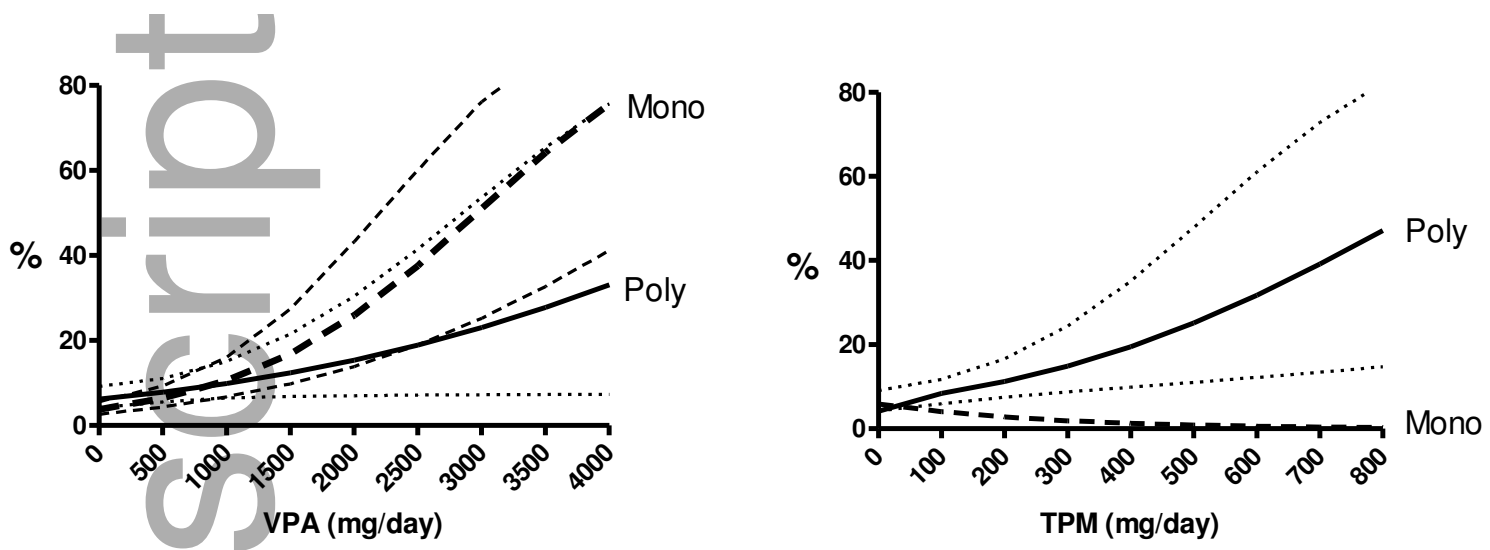

Fig 2. Logistic regressions and their $95 \%$ confidence intervals for risk of malformed pregnancy on dose of valproate (VPA) and topiramate (TPM) in polytherapy (in black) and monotherapy (in red) pregnancies, doses of the other AEDs involved in each case being held at their mean values. There was no statistically significant regression for topiramate monotherapy

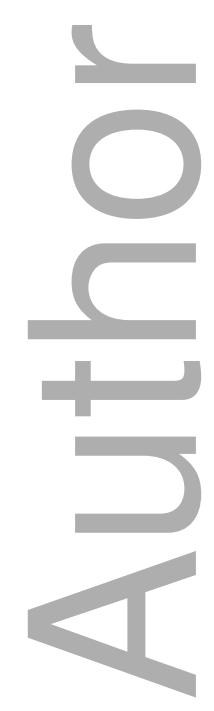


Table 1 . The more commonly employed pairs of antiepileptic drugs in all the 508 polytherapy pregnancies, and in the 416 polytherapy pregnancies that involved 2 drugs only. Abbreviations: $\mathrm{LTG}=$ lamotrigine, VPA=valproate, $\mathrm{LEV}=$ levetiracetam, $\mathrm{CBZ}=$ carbamazepine, $\mathrm{CZP}=$ clonazepam, $\mathrm{TPM}=$ topiramate .

\begin{tabular}{|c|c|c|c|c|}
\hline \multirow{2}{*}{ Combination } & \multicolumn{2}{|c|}{ All Polytherapy } & \multicolumn{2}{|c|}{ Two AED Polytherapy } \\
\hline & Number & $\%$ & Number & $\%$ \\
\hline $\mathrm{LTG}+\mathrm{VPA}$ & 104 & 20.5 & 79 & 19.0 \\
\hline $\mathrm{LTG}+\mathrm{CB}$ & 51 & 10.0 & 39 & 9.4 \\
\hline LTG + LEV & 50 & 9.8 & 36 & 8.7 \\
\hline $\mathrm{CBZ}+\mathrm{LEV}$ & 40 & 7.9 & 30 & 7.2 \\
\hline $\mathrm{LTG}+\mathrm{CZP}$ & 33 & 6.5 & 15 & 3.6 \\
\hline $\mathrm{CBZ}+\mathrm{TPM}$ & 30 & 5.9 & 13 & 3.1 \\
\hline $\mathrm{LTG}+\mathrm{TPM}$ & 28 & 5.5 & 18 & 4.3 \\
\hline $\mathrm{CBZ}+\mathrm{VPA}$ & 27 & 5.3 & 17 & 4.1 \\
\hline $\mathrm{CBZ}+\mathrm{CZP}$ & 26 & 5.1 & 14 & 3.4 \\
\hline $\mathrm{VPA}+\mathrm{CZP}$ & 26 & 5.1 & 13 & 3.1 \\
\hline $\mathrm{LEV}+\mathrm{TPM}$ & 25 & 4.9 & 14 & 3.4 \\
\hline $\mathrm{VPA}+\mathrm{LEV}$ & 25 & 4.9 & 10 & 2.4 \\
\hline
\end{tabular}


Table 2. Seizure freedom rates in the pre-pregnancy year and throughout pregnancy and labour for women with focal and generalised epilepsy who were managed with AED monotherapy or polytherapy. Seizure freedom rates were statistically significantly lower for the polytherapy pregnancies that the monotherapy pregnancies in all 4 comparisons

\begin{tabular}{|c|c|c|c|c|}
\hline Epilepsy type & \multicolumn{2}{|c|}{ Focal } & \multicolumn{2}{|c|}{ Generalised } \\
\hline Number $(\%)$ & \multicolumn{2}{|c|}{$887(53.1 \%)$} & \multicolumn{2}{|c|}{$784(46.9 \%)$} \\
\hline Treatment & Polytherapy & Monotherapy & Polytherapy & Monotherapy \\
\hline \multicolumn{5}{|c|}{ Throughout pre-pregnancy year } \\
\hline Number & 267 & 619 & 214 & 570 \\
\hline Seizure-fre & 105 & 349 & 97 & 399 \\
\hline Percent & 39.3 & 56.9 & 49.3 & 70.7 \\
\hline \multicolumn{5}{|c|}{ Throughout pregnancy and labour } \\
\hline Seizure-free & 96 & 318 & 88 & 391 \\
\hline Percent & 36.0 & 51.9 & 41.1 & 69.3 \\
\hline
\end{tabular}


Table 3. Intercept values and coefficients (b) for logistic regressions: logit risk $=a+b_{1} \cdot x_{1}+$ $\mathrm{b}_{2} \cdot \mathrm{x}_{2}+\ldots b_{\mathrm{n}} \cdot \mathrm{x}_{\mathrm{n}}$, where $\mathrm{b}$ refers to each individual AED and $\mathrm{x}$ represents its dose.

\begin{tabular}{lcccc}
\hline & \multicolumn{2}{c}{ Polytherapy } & \multicolumn{2}{c}{ Monotherapy } \\
& & $\mathrm{P}=$ & $\mathrm{P}=$ \\
\hline Intercept value & -2.256599 & $<0.0001$ & -2.90731 & $<0.0001$ \\
Coefficients & & & & \\
Carbamazepine & -0.000689 & 0.1393 & +0.000537 & 0.1620 \\
Valproate & +0.000502 & 0.0491 & +0.001093 & $<0.0001$ \\
Lamotrigine & -0.002458 & 0.0293 & -0.000971 & 0.3733 \\
Levetiracetam & +0.000042 & 0.7957 & -0.000484 & 0.2178 \\
Topiramate & +0.003257 & 0.0042 & -0.003952 & 0.4301 \\
Phenytoin & -0.000948 & 0.6376 & -0.002462 & 0.4365 \\
Oxcarbazepine & -0.000717 & 0.6075 & +0.000603 & 0.4171 \\
Clonazepam & -0.099615 & 0.6397 & - & - \\
\hline
\end{tabular}


Table 4: Percentages for (i) foetal malformation rates and (ii) seizure-free pregnancies in the (a) pre-pregnancy year and (b) during pregnancy in all polytherapy pregnancies and in polytherapy pregnancies involving 2 AEDs only. Numbers of relevant pregnancies are shown in Table 1.

\begin{tabular}{lcccccc}
\hline & \multicolumn{3}{c}{ All Pregnancies } & \multicolumn{2}{c}{ Pregnancies with 2 AEDs only } \\
Combination & Malformed & Seizure free & Malformed & \multicolumn{2}{c}{ Seizure free } \\
& & Before & During & & Before & During \\
\hline LTG + VPA & 8 & 46 & 41 & 10 & 53 & 43 \\
LTG + CBZ & 6 & 33 & 33 & 5 & 33 & 33 \\
LTG + LEV & $\mathbf{0}$ & $\mathbf{5 0}$ & $\mathbf{4 6}$ & $\mathbf{0}$ & $\mathbf{5 3}$ & $\mathbf{4 7}$ \\
CBZ + LEV & 5 & 43 & 30 & 7 & 44 & 30 \\
LTG + CZP & 3 & 21 & 18 & 7 & 27 & 27 \\
CBZ + TPM & 10 & 27 & 20 & 23 & 46 & 46 \\
LTG + TPM & 7 & 18 & 21 & 11 & 22 & 22 \\
CBZ + VPA & 15 & 33 & 22 & 23 & 35 & 24 \\
CBZ + CZP & 0 & 35 & 35 & 0 & 50 & 50 \\
VPA + CZP & 12 & 39 & 35 & 23 & 54 & 31 \\
LEV + TPM & 0 & 28 & 36 & 0 & 29 & 43 \\
VPA + LEV & 20 & 28 & 20 & 40 & 30 & 20 \\
\hline
\end{tabular}



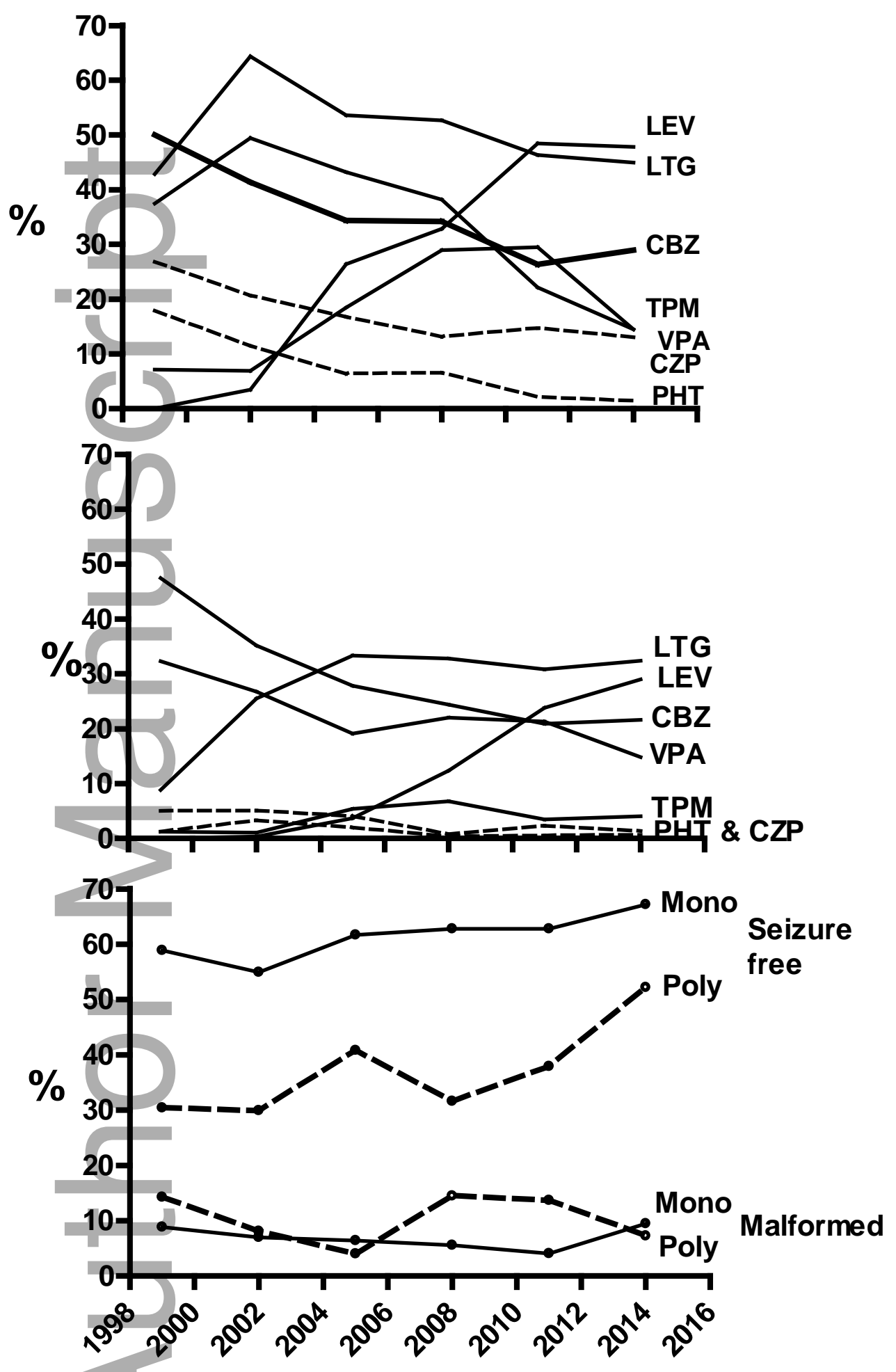


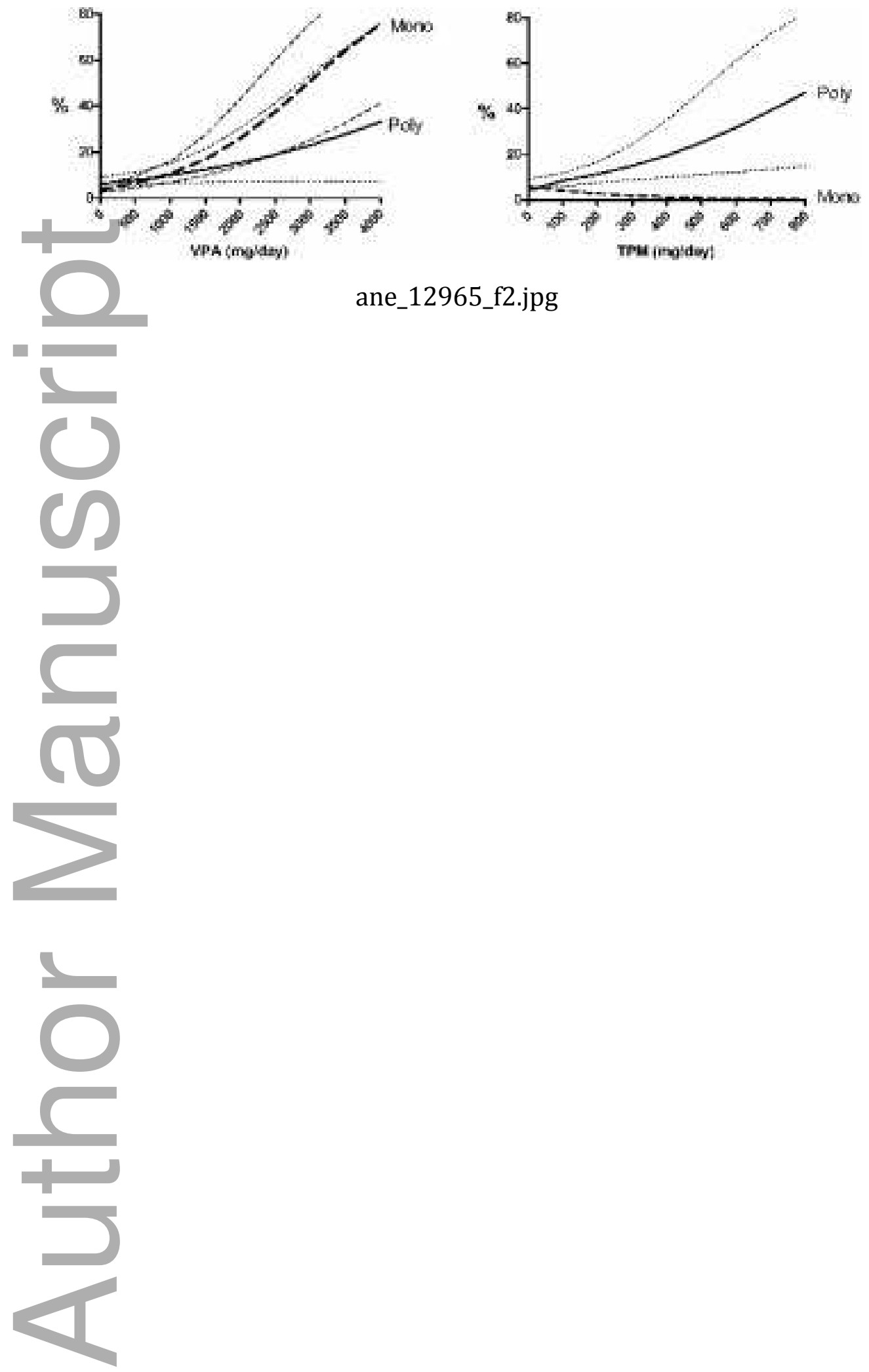

This article is protected by copyright. All rights reserved 


\section{University Library}

\section{- M M N E R VA A gateway to Melbourne's research publications}

Minerva Access is the Institutional Repository of The University of Melbourne

Author/s:

Vajda, FJE;O'Brien, TJ;Graham, JE;Hitchcock, AA;Lander, CM;Eadie, MJ

Title:

Antiepileptic drug polytherapy in pregnant women with epilepsy

Date:

2018-08-01

Citation:

Vajda, F. J. E., O'Brien, T. J., Graham, J. E., Hitchcock, A. A., Lander, C. M. \& Eadie, M. J. (2018). Antiepileptic drug polytherapy in pregnant women with epilepsy. ACTA NEUROLOGICA SCANDINAVICA, 138 (2), pp.115-121. https://doi.org/10.1111/ane.12965.

Persistent Link:

http://hdl.handle.net/11343/283989 